$$
\begin{aligned}
& \lambda=\lambda_{0}^{\prime} \tau+1 / \lambda_{2} \lambda_{0}{ }_{0} \tau^{2} \quad \beta=1 / 2 \beta_{0}^{\prime \prime} \tau^{2} \\
& \sin \lambda=\lambda_{0}^{\prime} \tau+1 / 2 \lambda_{0}^{\prime \prime} \tau^{2} \quad \sin \beta=1 / 2 \beta_{0}^{\prime \prime} \tau^{2} \\
& \cos \lambda=\mathrm{r}-1 / 2 \lambda_{0}^{2} \boldsymbol{\tau}^{2} \\
& \cos \beta=1 \text {. }
\end{aligned}
$$

Die Differentialformeln erhalten wir nach $T h . u$. Oppolser (Lehrbuch zur Bahnbestimmung Bd. II p. 430-434) wie folgt:

$\Delta \cdot \cos \beta \mathrm{d} \lambda=\mathrm{d} y_{0}+\boldsymbol{r}\left(\mathrm{d} y_{0}^{\prime}-\lambda_{0}^{\prime} \mathrm{d} x_{0}\right)+\boldsymbol{r}^{2}\left(-\lambda_{0}^{\prime} \mathrm{d} x_{0}^{\prime}+\left[3 x_{0} y_{0} /\left(2 r_{0}^{5}\right)-1 / 2 \lambda_{0}^{\prime \prime}\right] \mathrm{d} x_{0}+\left[3 y_{0}^{2} /\left(2 r_{0}^{5}\right)-1 / 2 \lambda_{0}^{\prime}{ }^{2}\right] \mathrm{d} y_{0}\right)$

$\Delta \cdot \mathrm{d} \beta=\mathrm{d} z_{0}+\tau \mathrm{d} z_{0}^{\prime}+\tau^{2}\left(\left[3 x_{0} z_{0} /\left(2 r_{0}^{5}\right)-1 / 2 \beta_{0}^{\prime \prime}\right] \mathrm{d} x_{0}+\left[3 y_{0} z_{0} /\left(2 r_{0}^{5}\right)\right] \mathrm{d} y_{0}+\left[3 z_{0}^{2} /\left(2 r_{0}^{5}\right)-\mathrm{r} /\left(2 r_{0}{ }^{3}\right)\right] \mathrm{d} z_{0}\right)$ und drei für die zweite Koordinate.

Aus diesen Gleichungen lassen sich die Unbekannten auf folgende Weise bestimmen.

Fïr $\tau_{0}=0$ bleibt in der ersten Gleichung nur $d y_{0}$ und in der zweiten nur $\mathrm{d} z_{0}$, welche Größen somit bestimmt sind. Aus den uibrigen $z$ wei Gleichungen für $\mathrm{d} \beta$ erhalten wir $\mathrm{d} z_{0}^{\prime}$ und $\mathrm{d} x_{10}$. Aus den zwei Gleichungen für $\mathrm{d} \lambda$ erhalten wir $\mathrm{d} y_{0}^{\prime}$ und $\mathrm{d} x_{0}^{\prime}$.

Sind $\tau_{1}$ und $\tau_{2}$, folglich auch $\tau_{1}{ }^{2}$ und $r_{2}{ }^{2}$, sehr klein, so sehen wir, daß vier Unbekannte $\mathrm{d} z_{0}^{\prime}, \mathrm{d} x_{0}, \mathrm{~d} y_{0}^{\prime}$ und $\mathrm{d} x_{0}^{\prime}$ nicht sicher bestimmt werden können. Sind aber nur $\boldsymbol{r}_{1}{ }^{2}$ und $\pi_{2}{ }^{2}$ klein, so lassen sich nur $\mathrm{d} x_{0}$ und $\mathrm{d} x_{0}^{\prime}$ nicht genau bestimmen. Sind aber $x_{1}{ }^{2}$ und $\tau_{2}{ }^{2}$ nicht sehr klein, so können doch Fälle eintreten, wo $\mathrm{d} x_{0}$ und $\mathrm{d} x_{0}^{\prime}$ sich aus diesen Gleichungen nicht bestimmen lassen, nämlich wenn die Koeffizienten $C=3 x_{0} y_{0} /\left(2 r_{0}^{5}\right)-1 / 2 \beta_{0}^{\prime \prime}$ bei $\mathrm{d} x_{0}$ und $\lambda_{0}^{\prime}$ bei $\mathrm{d} x_{0}^{\prime}$ gleich Null oder sehr klein sind.

Die erste Größe werden wir transformieren. Aus den bekannten Gleichungen

$$
\begin{gathered}
x-X=\Delta \cos \lambda \cos \beta \\
z-Z=\Delta \sin \beta
\end{gathered}=\Delta \sin \lambda \cos \beta
$$

erhalten wir für $t=t_{0}$ :

$$
x_{0}-X_{0}=\Delta_{0} \quad y_{0}-Y_{0}=0 \quad z_{0}-Z_{0}=0
$$

also ist $\Delta_{0}$ nach der $x$-Achse gerichtet und $z_{0}=Z_{0}$. Nach zweimaligem Differentiieren erhalten wir für $t=t_{0}$ :

$$
z^{\prime \prime}{ }_{0}-Z^{\prime \prime}{ }_{0}=\Delta_{0} \beta^{\prime \prime}{ }_{0} \text {. }
$$

Aus den Bewegungsgleichungen haben wir:

$$
\begin{gathered}
z_{0}^{\prime \prime}=-z_{0} / r_{0}{ }^{3} \quad Z_{0}^{\prime \prime}=-Z_{0} / R_{0}{ }^{3}=-z_{0} / R_{0}{ }^{3} \\
\beta_{0}^{\prime \prime}=z_{0}\left(\mathrm{I} / R_{0}{ }^{3}-\mathrm{I} / r_{0}{ }^{3}\right) / \Delta_{0} .
\end{gathered}
$$

Außerdem ist:

$$
x_{0} / r_{0}=\cos \left(X r_{0}\right)=\cos \left(\Delta_{0} r_{0}\right)
$$

Jekaterinoslaw, Berginstitut, I 9 × 4 Juli. tind folglich :

$$
R_{0}^{2}=\Delta_{0}^{2}+r_{0}^{2}-2 \Delta_{0} r_{0} \cos \left(\Delta_{0} r_{0}\right)
$$

$$
\cos \left(\Delta_{0} r_{0}\right)=x_{0} / r_{0}=\left(\Delta_{0}{ }^{2}+r_{0}{ }^{2}-R_{0}^{2}\right) /\left(2 \Delta_{0} r_{0}\right) .
$$

Setzen wir diese Werte in die gesuchte Größę und führen die Bezeichnungen $\Delta_{0} / R_{0}=\zeta, r_{0} / R_{0}=\eta$ ein, so erhalten wir

$$
C={ }_{3} R_{0}{ }^{2} z_{0}\left(\zeta^{2}-{ }_{\mathrm{I}}+5 / 3 \eta^{2}-2 /{ }_{3} \eta^{5}\right) /\left(4 \Delta_{0} r_{0}{ }^{5}\right) \text {. }
$$

Diese Größe verschwindet für $z_{0}=Z_{0}=0$ und für $\zeta^{2}=\mathrm{I}-\overline{5} /{ }_{3} \eta^{2}+{ }^{2} / 3 \eta^{5}$. Im ersten Falle liegt die Sonne in unserer geozentrischen $X Y$-Ebene, welche durch die relative (zur Erde) Geschwindigkeit des Himmelskörpers geht. Liegen also Erde, Sonne, Himmelskörper und die relative Geschwindigkeit in einer Ebene, so ist $C=$ o. Im zweiten Falle bestimmt die Gleichung eine Rotationsfläche mit der Achse Erde-Sonne. $\mathrm{Zu}$ dieser Fläche ist Herr Charlier gekommen bei einer ähnlichen Aufgabe $\left.{ }^{1}\right)$. Liegt also der Himmelskörper auf dieser Fläche, so verschwindet $C$. In beiden Fällen kann also die Unbekannte $\mathrm{d} x_{0}$ aus unseren Gleichungen nicht bestimmt werden, und wir müssen $\mathrm{d} x_{0}$ aus den Gliedern dritter und vierter Ordnung berechnen. Dazu ist noch zu bemerken, daß wir im Falle gleicher Zwischenzeiten, $\tau_{2}=$ $-\tau_{1}$, auch nicht mit Gliedern dritter Ordnung auskommen, $\mathrm{da}$ die Koeffizienten bei $\mathrm{d} s_{0}^{\prime}$ für $\tau_{1}$ und $\tau_{2}$ gleich und mit umgekehrtem Zeichen, ebenso wie bei $d x_{0}$, sind. Also muß $\mathrm{d} x_{0}$ mit Hilfe der Glieder mindestens vierter Ordnung bestimmt werden.

Bei dem Kometen I 900 III haben wir gerade diesen Fall. Es ist nämlich $z_{0}$ klein und der Komet war nicht weit von der Rotationsfläche gewesen. Dieser Umstand hat sich schon bei den Berechnungen der vorläufigen Bahn von Herrn Giacobini und Herrn Abold in der Weise gezeigt, daß die erhaltenen Elemente aus einer $Z$ wischenzeit von etwa $5^{\circ}$ Tagen ziemlich verschieden herauskamen.

S. Scharbe.

$\left.{ }^{2}\right)$ C. $I$. L. Charlier. Die analytische Lösung des Bahnbestimmungsproblems. Arkiv för Matematik, Astronomi och Fysik, Bd. 7 , No. Io, pg. 27 .

\title{
Bemerkungen zu fünf einander benachbarten Sternen der BD.
}

Bei der Vergleichung der Bonner Karte der Gegend meiner hypothetischen Ephemeride für den Planeten I 9 I 3 e in A. N. $475 \mathrm{I}$ mit dem Himmel fand ich folgende Unterschiede:

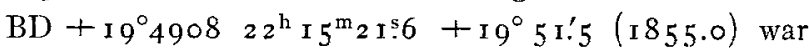
I 9 I 4 Juli 19 und Aug. I 9 am Kartenort nicht sichtbar. Einen in der gleichen AR. südlicher stehenden Stern verglich ich Aug. 29 am Ringmikrometer des hiesigen Refraktors mit dem gleichhellen $9^{\mathrm{m}} \cdot 4$ Berl A $9 \mathrm{I}_{5} 8$, welcher in $\mathrm{BD} 9^{\mathrm{m}} .0$ angegeben ist, und fand r $914.022^{\mathrm{h}}{ }_{1} 8^{\mathrm{m}} \mathrm{II} .05+20^{\circ} \mathrm{O}^{\prime} \mathrm{I} 8^{\prime \prime} .3$, sodaß der Kartenort $1855.022^{\mathrm{h}} 15^{\mathrm{m}} 22^{\mathrm{s}} .2+19^{\circ} 42^{\prime} .6$ sein würde.

$\mathrm{BD}+19^{\circ} 491822^{\mathrm{h}} \mathrm{I} 8^{\mathrm{m}} \mathrm{I} \mathrm{I}^{\mathrm{s}} \mathrm{I}+19^{\circ} 50^{\prime} 6$ vermißte ich am Himmel r9 14 Juli I9, sowie Aug. I9 und 22.
$\mathrm{BD}+19^{\circ} 49_{21} 22^{\mathrm{h}} 19^{\mathrm{m}} \mathrm{I}^{\mathrm{s}} 6{ }^{\circ}+\mathrm{I} 9^{\circ} 37^{\prime} \cdot 3$ vermißte ich am Himmel I 9 I 4 Aug. 24 und 28.

$\mathrm{BD}+20^{\circ} 5^{\mathrm{I}} 7022^{\mathrm{h}} 23^{\mathrm{m}} 8^{\mathrm{s}} \cdot 5+20^{\circ} 40^{\prime} \cdot 9$ vermißte ich am Himmel I9 I 4 Juli I9, sowie Aug. I9 und 22 .

$\mathrm{BD}+20^{\circ} 5^{\mathrm{I}} 7922^{\mathrm{h}} 25^{\mathrm{m}} 27^{\mathrm{s}} 3+20^{\circ} 44^{\prime} \mathrm{o}$. In nächster Nähe dieses Ortes stehen jetzt am Himmel zwei schwache Sterne $\mathrm{I} \mathrm{I}^{\mathrm{m}}$, welche einander $5^{8}$ auf dem Parallel folgen. Nicht weit davon steht ein etwas hellerer, etwa $10^{\mathrm{m}}$, dessen Ort ich Aug. 30 durch Vergleichung mit Berl B $865^{2}$ so bestimmte: I 9 I $4.022^{\mathrm{h}} 28^{\mathrm{m}} 22^{\mathrm{s}} \cdot 5^{2}+20^{\circ} 57^{\prime} 3 \mathrm{II}^{\prime \prime} \cdot 2, \quad$ I $855.0 \quad 22^{\mathrm{h}} 25^{\mathrm{m}} 33^{\mathrm{s}}$. I $+20^{\circ} 39: 4$.

Düsseldorf, Sternwarte, I 9 I 4 Sept. 3. Wilhelm Luther. 
Nach Prüfung der Originale der BD bemerkt Geheimrat Küstner über diese Sterne folgendes:

Die vermißten 5 Sterne der BD sind in folgenden Sucherzonen beobachtet:

S.-Z. $480, K r$. I 854 Juli 25 , Luft sehr dunstig.

S.-Z. $520, K r .1854$ Sept. 2 I, meistens sehr dunstig.

S.-Z. 559, Sch. I 854 Okt. 28 , gute Luft, $\mathbb{C}$ im I. Viertel.

Die einzelnen Beobachtungen sind:

$+19^{\circ} 4908$ S. $-Z .48022^{\mathrm{h}}{ }_{1} 5^{\mathrm{m}} 22^{\mathrm{s}} \cdot 7+19^{\circ} 50^{\prime} 2$ Teilstrich undeutlich! D20 $20.5 \quad 52.9$ Teilstrich - I.2 deutlich.

Hier wäre also eine Korrektion von - r Teil $=-7^{\prime}$ nicht ausgeschlossen; das eine $\mathrm{Mal}$ ist der Teilstrich undeutlich, das andere Mal könnte der Beobachter statt - $\mathbf{2 . 2}$ gelesen haben - $-\mathbf{I} .2$.
+ I $9^{\circ} 4918$ S.-Z. $48022^{\mathrm{h}}{ }_{1} 8^{\mathrm{m}}{ }_{\mathrm{II}} \mathrm{s} .7+\mathrm{I} 9^{\circ} 48: 8$ | alles deutlich

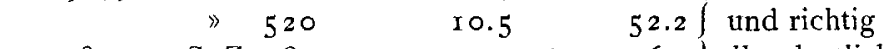
+ I $9^{\circ} 492$ I S.-Z. 48022 I 92.7 + 9936.3 alles deutlich $\left.\begin{array}{lll}520 & 0.5 & 38.3\end{array}\right\}$ und richtig $+20^{\circ} 5$ I 70 S. $-Z .5202223 \quad 7.5+2041.5$ alles deutlich

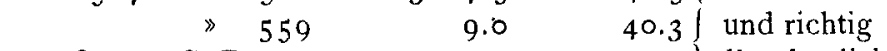
$+20^{\circ} 5$ I 79 S. Z. 520222523.5 +20 45.0 alles deutlich 2 $559 \quad 30.0 \quad 43.0 /$ und richtig (Größen durchweg $\circ=9^{\mathrm{m}} \cdot 5$.)

Diese 4 Sterne erscheinen durchaus gesichert, und es wäre doch sehr merkwürdig, wenn sie jetzt alle am Himmel fehlen oder veränderlich sein sollten. Von den beiden schwachen Sternen am Ort von $+20^{\circ} 5179$ wird wohl in $B D$ das vereinte Licht undeutlich beobachtet sein; man kann nicht wohl schließen, daß einmal der eine und das andere Mal der andere beobachtet ist.

Bonn, K. Sternwarte, Igr4 Sept. 3. F. Küstner.

\section{Die geschichtete Linienemission.}

Spektralaufnahmen des Dumbbellnebels $\mathrm{M} 27$ (NGC) 6853) unter verschiedenen Positionswinkeln, wobei also der Spalt des Spektrographen den Nebel unter verschiedenen Richtungen durchschneidet, zeigen, daß im Dumbbellnebel eine analoge Gesetzmäßigkeit in der Verteilung der Gase herrscht, wie ich sie beim Ringnebel in der Leier ( $M{ }_{57}$ ) nachgewiesen habe.

Ein Querschnitt von Nord nach Süd gibt beispielsweise im allgemeinen in jeder Emissionslinie zwei Maxima, eines im Norden, das zweite im Suden. Sie sind durch ein Minimum, das von dem matteren Raum im innersten Teile des Nebels herrührt, unterbrochen. Die beiden Maxima liegen bei den verschiedenen Spektrallinien verschieden weit voneinander. Wieder sind am charakteristischsten die beiden Gase, die durch die Emissionen $\lambda_{373}$ und $\lambda_{469}$ repräsentiert werden. In der Linie $\lambda_{373}$ stehen die zwei Maxima am weitesten voneinander, also am weitesten außen. Umgekehrt hat $\lambda_{4} 69 \mathrm{im}$ Zentrum des Nebelflecks, dort wo $\lambda_{373}$ ihr Minimum besitzt, ibre Maxima.

Ein Querschnitt von Osten nach Westen zeigt das Maximum von $\lambda_{373} \mathrm{im}$ Westen, das Maximum von $\lambda_{4} 69$ im östlichen Teil des Nebelflecks und nahe der Mitte.

Kleinere Verschiedenheiten lassen die anderen Linien erkennen, und die gleichen Erscheinungen zeigt ein Querschnitt von Nordost nach Südwest.

Es ist somit die geschichtete und gegensätzliche Verteilung verschiedener Gase keine besondere Eigentümlichkeit des Ringnebels, sondern sie herrscht ebenso im Dumbbellnebel.

Heidelberg, Königstuhl-Sternwarte, I 914 Okt. 2.3. Max itolf.

\section{Osservazioni della cometa 1914 e (Campbell)}

all'equatoriale del $R$. Osservatorio astronomico al Collegio Romano.

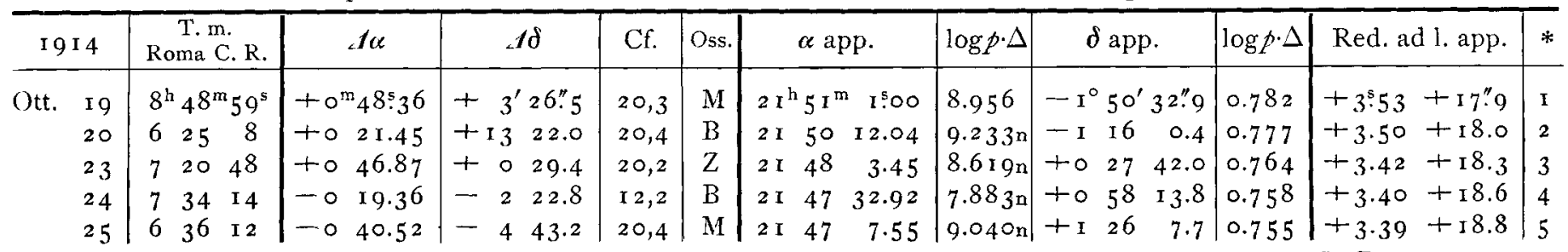

Ott. I9. Nucleo $\mathrm{xo}^{\mathrm{m}}$, testa $\mathrm{I}^{\prime}$. - Osservatore: $\mathrm{B}=$ E. Bianchi, $\mathrm{M}=$ E. Millosevich, $\mathrm{Z}=G . Z a p p a$. Stelle di confronto.

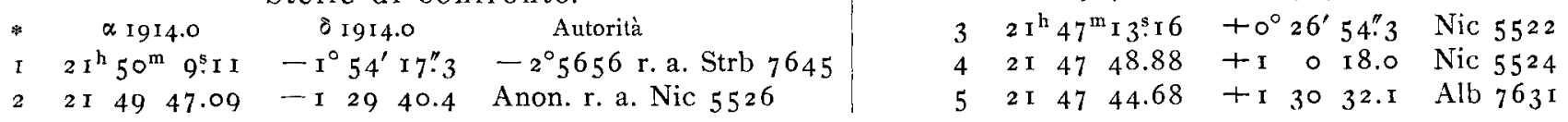

R. Osservatorio al Collegio Romano, Roma, I9 4 Ott. 27.

E. Millosevich.

Inhalt zu Nr. 477I. 7. Hartmann. Die Bewegung der elf hellsten Plejadensterne. 305. - G. Demetrescu. Sur la réfraction horizontale dans le calcul d'une éclipse. 309. - S. Kasakow. Zur Vergleichung der Planeten- und Kometenephemeriden mit den Beobachtungen. 3 I I. - S. Scharbe. Besondere Fälle bei definitiven Bahnbestimmungen aus einer Erscheinung. 3 I5. - Bemerkungen zu fünf einander benachbarten Sternen der BD. 3I7. - M. Wolf. Die geschichtete Linienemission. 319. E. Millosevich. Osservazioni della cometa 1914 e (Campbell). 319. 